SECTION 7. Mechanics and machine construction.

Sagat Zhunisbekov

doctor of technical Sciences, Professor, academician of the National Engineering Academy of the Republic of Kazakhstan, rector

Taraz technical Institute, Kazakhstan tar-ti@mail.ru

Alexandr Nikolayevich Shevtsov candidate of technical Sciences, President of International Academy International Academy of Theoretical \& Applied Sciences, (USA, Sweden, Kazakhstan)

Shev_AlexXXXX@mail.ru Zhanara Askerbayevna Keulimzhayeva 2 year Magistr, the specialty "Mathematics " Taraz State University named after M.Kh. Dulati, Kazakhstan Janka_9009@mail.ru

\title{
ON THE PROBLEM OF MODELING OF MACROMOLECULES OF POLYMERS
}

Abstract: In this article a number of studies and some aspects of construction of mathematical models of polymer molecules, their characteristics, properties and laws.

Key words: macromolecule, model, a polymer.

\section{О ПРОБЛЕМЕ МОДЕЛИРОВАНИЯ МАКРОМОЛЕКУЛ ПОЛИМЕРОВ}

Аннотация: В данной статье рассматривается ряд исследований и некоторые аспекты построения математических моделей полимерных молекул, их особенности, свойства и закономерности.

Ключевые слова: макромолекула, модель, полимер.

Одно из важных направлений развития теории полимерных макромолекул связанно с численными методами имитации молекулярных движений. Впервые исследования полимерной динамики проводились Штокмайером и Вердье, для достаточно простых решеточных моделей полимерных цепей (Рис.1) методом МонтеКарло [1, 2]. Данный метод предполагал задание типов элементарных перестроек конформаций цепи, а затем разыгрывалось временное изменение ансабля цепей. В последующем, метод Монте-Карло был развит и углублен в работах Жени и Моннери, Тарана, Гриднева, Скворцова, Бирштейна и Готлиба и др.

В настоящее время наиболее применяемым и дающим наиболее детальное описание макромолекулярной динамики с учетом микроструктуры и локальных взаимодействий дает прямая имитация молекулярного движения методами молекулярной и броуновской динамики.

В методе Молекулярной динамики решаются уравнения классической механики для малого объема полимерной системы, содержащего частицы цепей и растворителя.Сам метод основан на специальных математических методах молекулярной динамике цепных молекул с жесткими связями в работах Балабаева, Гривцова и Шноля, Даринского, Готлиба, Неелова, Лукьянова, Олейника, Мазо, Риккерта, Гунстерена, МакКаммона и др.. 


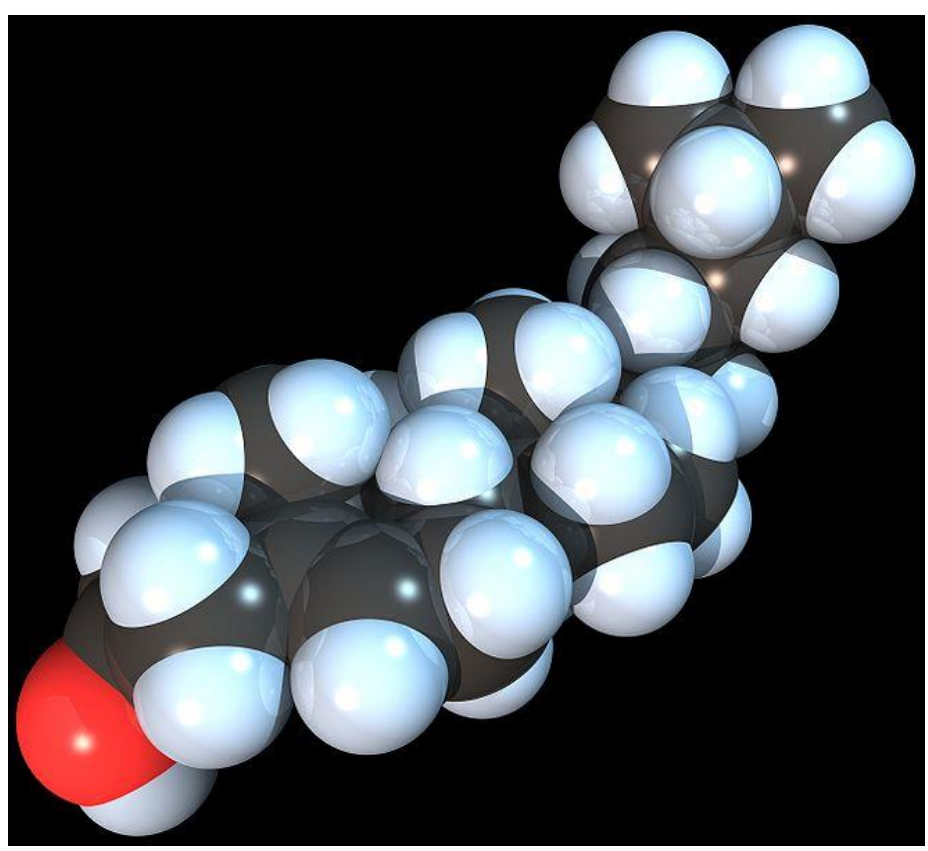

Рисунок 1 - Модель полимера [4].

Метод Броуновской динамики основан не на уравнениях движения выведенных в классической динамике, а на уравнениях Ланжевена для выделенной цепи на фоне вязкой матрицы, моделирующей окружающую среду (другие макромолекулы). Развитию этого методы посвященны труды Зимма, Фиксмана, Гельфанда, Даринского, Неелова, Клушина, Готлиба.

Методы прямой имитации молекулярного движения в отличие от численных мотодов моделирования на решетчатых моделях цепи методами Монте-Карло позволяет детально изучить механизмы локальной подвижности в полимерах без априорных предпочтений о типе, характере и масштабах элементарных движений, характерных другим методам.

\section{Пространственные модели и проблема аппроксимации.}

Одной из основных проблем изучаемой при исследовании макромолекул, и различных полимерных структур, является - проблема разработки адекватного и эффективного алгоритма апроксимации структуры макромолекулы - более простыми аналогами.

Для сравнительной оценки гибкости макромолекул различных полимеров их цепи представляют свободно-сочленёнными, состоящими из отрезков, называемых статистическими сегментами. Каждый из сегментов включает столько мономерных звеньев, сколько необходимо для того, чтобы он мог свободно вращаться относительно предыдущего сегмента (рис.2). 


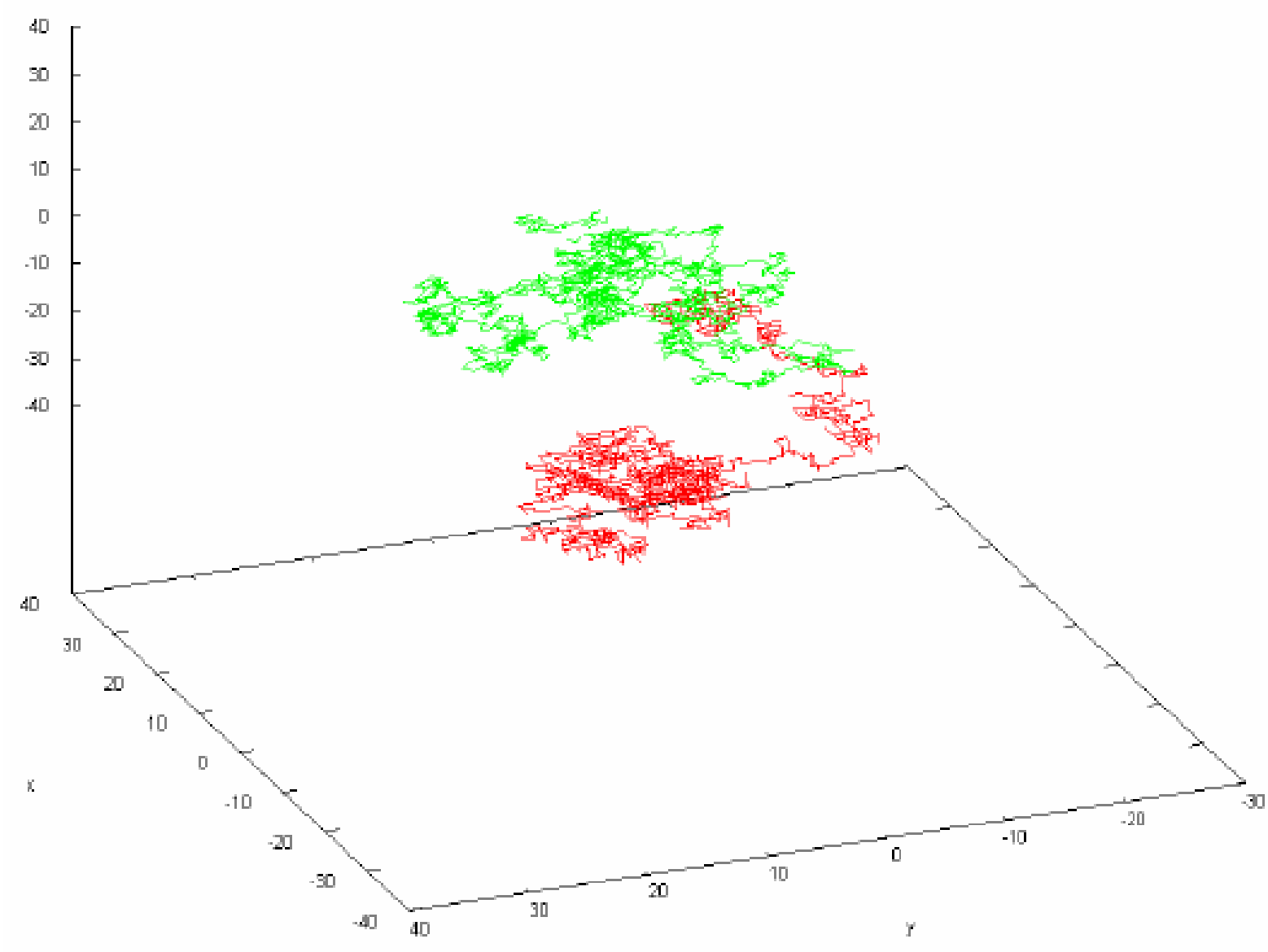

Рисунок 2 - Пространственные модели макромолекул.

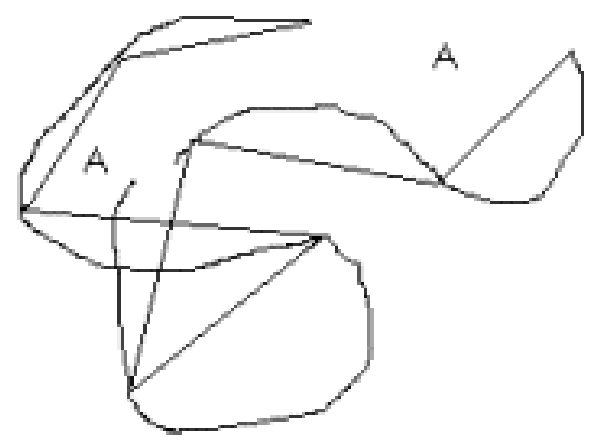

Рисунок 3 - Полимерная цепь, образованная из статистических сегментов Куна.

Таким образом, статистический сегмент (или как его ещё называют сегмент Куна) является мерой свободы вращения в реальных макромолекулах и, соответственно, мерой их гибкости. Учитывая сказанное, квадрат среднего расстояния между концами цепи $\bar{h}^{2}$ и её контурную длину L можно выразить через величину сегмента. Таким образом, в зависимости от гибкости полимерной цепи её сегмент может содержать от нескольких единиц до нескольких десятков мономерных звеньев. 


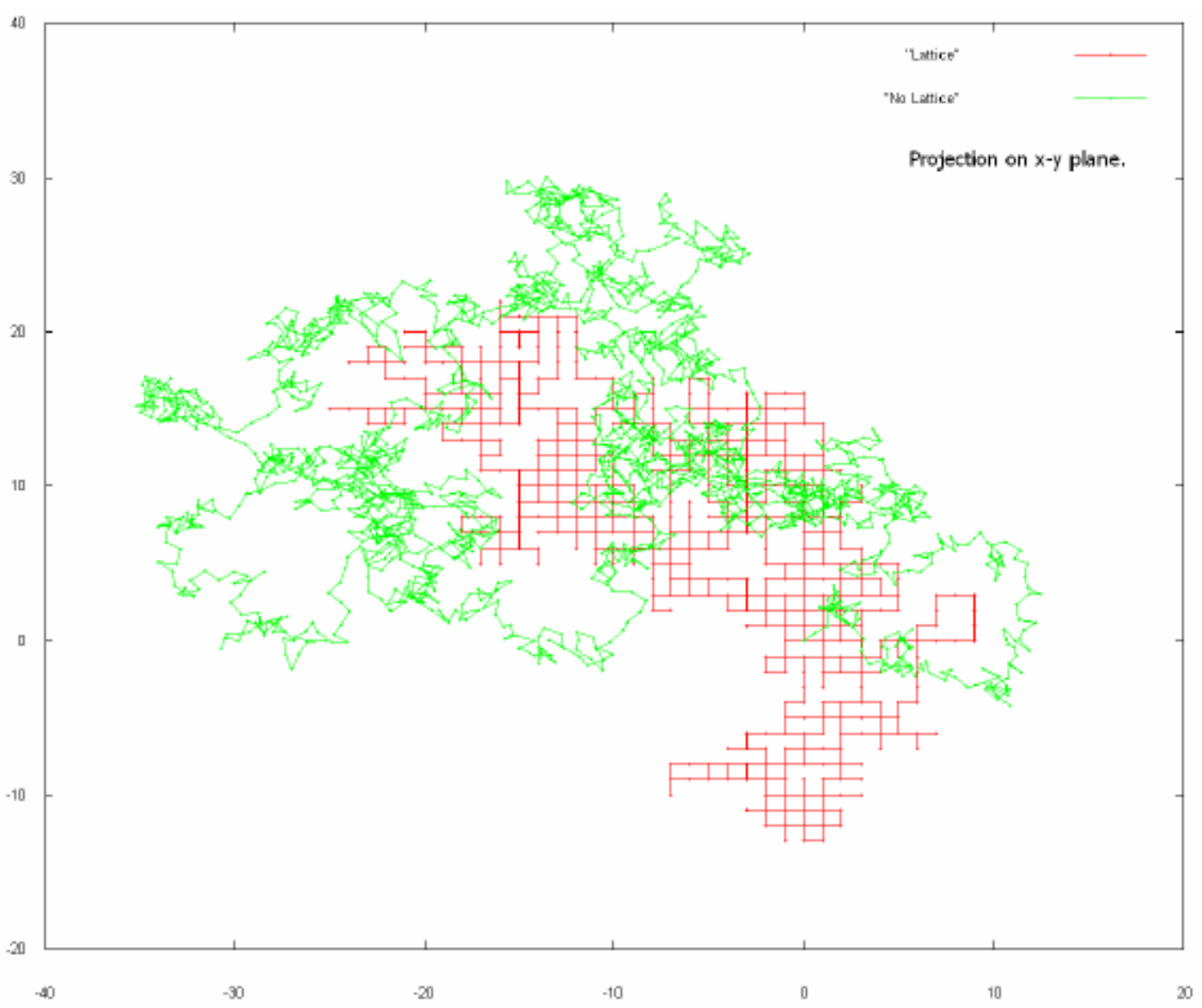

Рисунок 4 - Аппроксимация молекулы статистическими сегментами.

\section{Основные подходы к математическому моделированию макромолекул}

Основная задача статистической теории - вычисление средних значений различных величин, которые характеризуют поведение системы в состоянии равновесия[5]. Существуют два подхода к решению этой общей задачи. В первом случае среднее значение $\langle A>$ некоторого свойства $A(\mathrm{r}, \mathrm{v})$, которое предполагается зависящим от совокупности координат- $\{\mathrm{r}\}$ и скоростей $\{\mathrm{v}\}$ частиц, определяют путем усреднения множества "мгновенных" значений $A[\mathrm{r}(\mathrm{t}), \mathrm{v}(\mathrm{t})]$, наблюдаемых в последовательные моменты времени $t$ на достаточно протяженном интервале $\tau$ :

$$
A=\frac{1}{\tau} \int_{0}^{\tau} A[r(t), v(t)] d t
$$

Этот подход, называемый усреднением по времени, исходит из того, что нам известны законы движения частиц системы.

Альтернативный путь вычисления средних значений параметров системы был определен Больцманом, а затем развит Гиббсом в отдельную теорию. Идея этого подхода заключается в том, что наблюдаемое свойство рассматривается не как среднее по времени, а как среднее по множеству различных состояний системы, которые возникают с определенной вероятностью. Такой подход называют усреднением по 
ансамблю. Вероятность (или частота) возникновения того или иного состояния пропорциональна его статистическому весу

$$
\omega=\mathrm{e}^{-U / \mathrm{kT}},
$$

где $U$ - потенциальная энергия данной конфигурации,

$k$ - константа Больцмана,

$T$ - абсолютная температура.

В этом случае наблюдаемые средние значения даются общим выражением

$$
A=\int \ldots \int A(\mathrm{r}) \omega(\mathrm{r}) \mathrm{dr} / \int \ldots \int \omega(\mathrm{r}) \mathrm{dr}
$$

Оба фундаментальных принципа определения средних значений могут быть положены в основу вычислительных схем, реализуемых на компьютере. При этом необходимо знать лишь способ расчета потенциальной энергии системы как функции координат r. Результаты расчетов, какого - либо свойства одной системы вычисляемые по одному и другому пути должны совпадать при длительном времени наблюдения за системой в первом подходе и при очень большом числе испытаний во втором подходе.

\section{Проблема расчета гибкости цепи}

Различают два вида гибкости цепи: термодинамическую (статистическую) и кинетическую (динамическую). Первая является равновесной, она определяется химическим строением макромолекул и реализуется в результате теплового движения отрезков цепи. Макромолекулы в растворах участвуют в тепловом движении посредством макроброуновского и микроброуновского движения. В первом случае макромолекулы перемещаются как целое, во втором - перемещаются отдельные кинетически независимые отрезки цепи, называемые сегментами. Микроброуновское движение осуществляется за счет столкновений сегментов макромолекул с другими сегментами или молекулами растворителя. В каждый момент времени сегмент претерпевает множество столкновений. Как правило, результирующий момент силы не равен нулю, в результате сегмент движется в направлении результирующего момента, а вместе с ним в эту сторону выгибается макромолекула. Многократные изгибы макромолекулы, являющиеся следствием еe участия в тепловом молекулярнокинетическом движении, приводят к свертыванию макромолекулы в клубок. Кинетическая гибкость характеризует скорость конформационных переходов, т.е. время, необходимое для смены локальных конформаций. Существует два механизма термодинамической гибкости, один из которых применим для гибкоцепных полимеров, другой - для жесткоцепных.

\section{Проблема учета упругости при построении модели полимера.}

\section{Механическая модель молекулы полимера.}

Под моделью полимера будем понимать правила, описывающие взаимодействие частиц между собой и/или с внешними полями, то есть в формулировке вида и способа вычисления функции потенциальной энергии. 
В ряде задач удобно рассматривать молекулу не как электронно-ядерную систему, а как систему взаимодействующих атомов. Предполагается, что энергию взаимодействия можно разделить на ряд составляющих [5].

\section{1. Энергия деформации связи.}

При отклонении длин связей от их нормального значения возникает энергия деформация связи ( $\left.\mathrm{E}_{\mathrm{c}}\right)$. Обычно связь рассматривают как гармонический осциллятор. При этом можно предположить, что при малых деформациях энергия ковалентных связей подчиняется следующей зависимости.

$$
\mathrm{U}(\mathrm{R})=\mathrm{K} \times\left(\mathrm{R}-\mathrm{R}_{\mathrm{i}}\right)^{2}
$$

где К - силовая постоянная,

$\mathrm{R}$ - равновесная длина связи

$\mathrm{R}_{\mathrm{i}}$ - мгновенная длина связи

Иногда энергию взаимодействия связанных атомов описывают “мягким" Леннард-Джонсовским потенциалом.

\section{2. Энергия деформации углов.}

Для каждого атома в молекуле существуют некоторые идеальные углы, отклонение от которых требуют затрат энергии угловых деформаций (Еугл). Предполагается, что эта энергия аддитивна, причём при малых отклонениях справедлив закон Гука. [7]

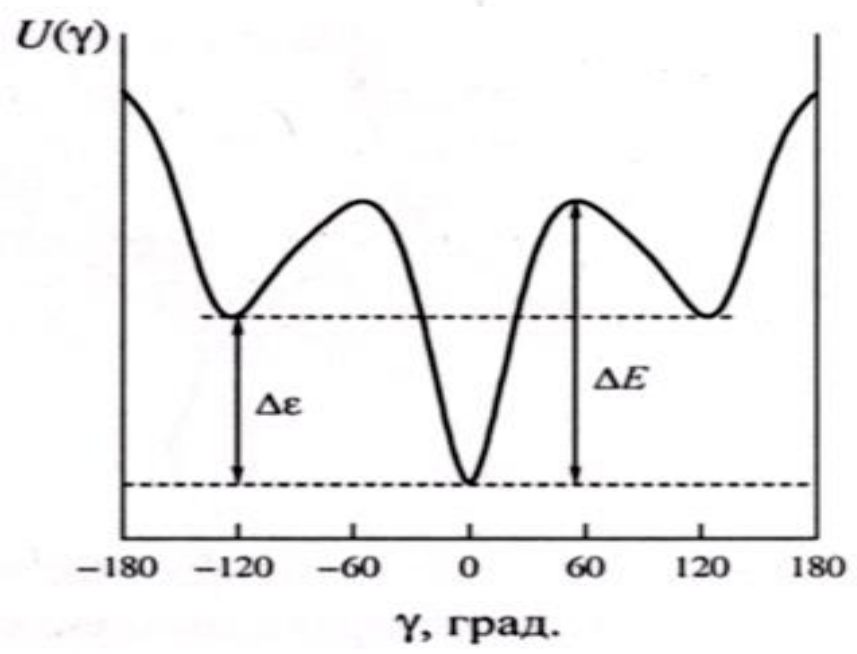

Рисунок 5 - Зависимость потенциальной энергии от угла поворота молекулы.

Аналогично энергии деформации связей можно записать.

$$
\mathrm{U}(\theta)=\mathrm{C} \times\left(\theta_{\mathrm{i}}-\theta\right)^{2}
$$

где С - силовая константа,

$\theta$ - идеальное значение угла связи,

$\theta_{\mathrm{i}}-$ мгновенное значение угла связи 
Деформация углов происходит значительно легче, чем деформация связей ( константа К на порядок выше, чем С).

\section{3. Невалентные взаимодействия атомов $\left(\mathbf{E}_{c}\right)$.}

Имеют ту же природу, что и межмолекулярные. Эти взаимодействия могут описываться различными потенциалами. Одним из примером описания является потенциал Леннарда-Джонса. Ван-дер-ваальсовые взаимодействия имеют тот же порядок, что и тепловое движение атомов (кТ). Если ещё учесть, что число этих взаимодействий равно $\mathrm{N}(\mathrm{N}-1) / 2$, то можно сделать вывод, что при согласованном действии этих сил могут происходить значительные крупномасштабные конформационные перестройки макромолекулы.

\section{4. Торсионная энергия.}

Потенциалы невалентных взаимодействий дают слишком малые значения барьеров внутреннего вращения. Это обстоятельство можно изменить, если в конформационную энергию ввести член, зависящий от взаимного расположения связей, присоединённых к оси вращения. Суммарные энергетические затраты, характеризующие отклонения от оптимальной взаимной ориентации связей, определяются как торсионная (илиориентационная( составляющая $\left(\mathrm{E}_{\mathrm{T}}\right)$ конформационной энергии.

$$
\mathrm{U}(\varphi)=\mathrm{U}_{0} / 2(1-\cos (\mathrm{n} \varphi))
$$

где $\mathrm{U}_{0}$ - барьер внутреннего вращения. Для карбоцепных полимеров это барьер составляет (между транс- и гош- состоянием) составляет примерно 3 ккал/моль.

n - порядок вращения. Для карбоцепных полимеров $\mathrm{n}=3$.

$\varphi$-значение двухгранного угла.

\section{5. Электростатические взаимодействия (Еэл).}

Важную роль играет для молекул содержащие сильно полярные связи. За счёт того, что более электроотрицательный атом оттягивает на себя электронную плотность возникает перераспределение заряда и появляются частично положительный и частично отрицательный заряд на атомах. То есть в ковалентная сильно полярная связь - это смесь ковалентной и ионной связи. Также электростатические взаимодействия проявляются в полиэлектролитах. За счёт ионизации ионогенных груп на полимерной цепочке возникают заряды, а следовательно электростатические взаимодействия. В этом случае эти взаимодействия являются дальнодействующими (убывают пропорционально 1/r) и определяют значительные крупномасштабные конформационные перестройки.

6. Другие составляющие энергии молекулы. 
Аналогично в выражении для конформационной энергии могут быть введены члены, учитывающие энергию водородных связей $\left(\mathrm{E}_{\mathrm{H}}\right)$, взаимодействие неподелённых электронных пар ( $\left.\mathrm{E}_{\mathrm{H}}\right)$, влияние среды ( $\left.\mathrm{E}_{\mathrm{P}}\right)$ и т.д.

Не все вклады одинаковы важны. Для каждого класса задач выбираются наиболее важные составляющие потенциальной энергии молекулы, а другие не учитываются.

\section{3. Методы молекулярной динамики (МД)}

Метод математического моделирования, основанный на подходе усреднения по времени наблюдения называют методом молекулярной динамики. Суть его состоит в следующем [5]:

Рассмотрим систему, состоящую из заданного числа частиц (атомов или молекул). В классической механике движение каждой частицы $\mathrm{i}$ с массой $\mathrm{m}_{\mathrm{i}}$ может быть описано уравнением Ньютона

$\mathrm{m}_{\mathrm{i}} \mathrm{a}_{\mathrm{i}}(\mathrm{t})=\mathrm{f}_{\mathrm{i}}(\mathrm{t}) \quad($ масса $\times$ ускорение $=$ сила $)$.

Здесь $\mathbf{f}_{\mathrm{i}}(\mathrm{t})$ - сила, действующая в данный момент времени $\mathrm{t}$ на частицу i со стороны всех остальных частиц системы (эта сила связана с потенциальной энергией известным соотношением $\left.\mathbf{f}_{\mathrm{i}}(\mathrm{t})=-\partial \mathrm{U} / \partial \mathbf{r}_{\mathrm{i}}\right) ;$ ускорение определяется как $\mathrm{a}_{\mathrm{i}}(\mathrm{t})=\mathrm{d} \mathbf{v}_{\mathrm{i}}(\mathrm{t}) / \mathrm{dt}$ или $\mathrm{a}_{\mathrm{i}}(\mathrm{t})=\mathrm{d}^{2} \mathbf{r}_{\mathrm{i}} / \mathrm{dt}^{2}$. Если эти производные заменить их конечно-разностными аналогами, то систему уравнений Ньютона, записанных для всех частиц, можно решить на компьютере. То есть, зная координаты частиц $\mathbf{r}(\mathrm{t})$ и отвечающие им силы $\mathbf{f}(\mathrm{t})$ в некоторый момент времени $t$, можно через небольшой промежуток времени $\Delta t$ найти новые координаты $\mathbf{r}(\mathrm{t}+\Delta \mathrm{t})$ и силы $\mathbf{f}(\mathrm{t}+\Delta \mathrm{t})$ в следующий момент времени $\mathrm{t}+\Delta \mathrm{t}$ и т.д., шаг за шагом. Очевидно, что скорости оцениваются как

$$
\mathbf{v} \approx[\mathrm{r}(\mathrm{t}+\Delta \mathrm{t})-\mathrm{r}(\mathrm{t})] / \Delta \mathrm{t}
$$

Вычисляя на каждом шаге интересующий нас параметр $A$ можно проследить его эволюцию во времени, а усреднив по достаточно большому числу сделанных шагов $s$ получаем искомые равновесные свойства. Такую схему расчета принято называть численным экспериментом динамического типа или просто методом молекулярной динамики (МД). Используются также различные вариации метода МД, в которых наряду с "внутренними" силами, обусловленными взаимодействием атомов друг с другом, включаются те или иные внешние силы. Подобные схемы моделирования составляют группу методов неравновесной молекулярной динамики.

\section{4. Метод Монте-Карло (МК)}

Вычислительную схему, в основе которой лежит альтернативный (вероятностный) принцип определения средних значения, называют методом статистических испытаний или методом Монте-Карло (МК) [5]. В этом методе переходы между состояниями системы осуществляются следующим образом. На каждом шаге случайным образом выбирается частица (или группа частиц) и перемещается на небольшое расстояние в случайном направлении. Это приводит к изменению потенциальной энергии системы на некоторую величину $\Delta U$, которая и определяет вероятность перехода 


$$
\mathrm{p} \sim \mathrm{e}^{-\Delta \mathrm{U} / \mathrm{kT}}
$$

из "старого" в "новое" состояние системы. Интересующие характеристики вычисляются на каждом шаге и усредняются по большому числу сделанных шагов.

\section{Термодинамические составляющие упругой силы}

Природа упругой силы, вызывающей обратимость деформаций, может быть выявлена при анализе термодинамики обратимого растяжения упругого тела [6]. В этом случае при условии $\mathrm{V}=$ const работа внешних сил равна

$$
f \cdot d l
$$

где $f$ - приложенная сила;

$d l$ - удлинение тела.

Согласно первому и второму закону термодинамики,

$$
f \cdot d l=d U-T d S
$$

откуда

$$
f=\left(\frac{\partial U}{\partial l}\right)_{T}-T\left(\frac{\partial S}{\partial l}\right)_{T}
$$

Таким образом, упругая сила имеет две составляющие. Первая из них $(\partial U / \partial I)_{\text {T }}$ соответствует силе, действие которой направлено на снижение внутренней энергии деформированного тела, вторая - $(\partial S / \partial I)_{T}$ направлена на повышение его энтропии.

Наиболее универсальной характеристикой упругости является модуль Юнга $E$ коэффициент пропорциональности закона Гука:

$$
\sigma=E \cdot \varepsilon,
$$

где $\sigma$ - напряжение; $\varepsilon$ - относительная деформация.

Ниже приведены значения модуля упругости ряда материалов и идеального газа в Па:

\begin{tabular}{|c|c|c|c|c|}
\hline Сталь & Медь & Кварц & Каучук & Газ \\
\hline $2 \cdot 10^{11}$ & $1 \cdot 10^{11}$ & $1 \cdot 10^{11}$ & $5 \cdot 10^{5}$ & $1 \cdot 10^{5}$ \\
\hline
\end{tabular}

По величине модуля упругости все приведенные материалы разбиваются на две группы, одну из которых составляют газы и каучуки, модуль которых намного порядков меньше по сравнению с металлами или стеклом (или в более общей форме по сравнению с кристаллами и переохлажденной застеклованной жидкостью), следовательно, их намного легче деформировать. Кроме того, оказалось, что 
температура принципиально различным образом влияет на модуль упругости двух различных групп материалов - нагревание приводит к уменьшению модуля упругости кристаллов, в том числе металлов, и увеличению модуля упругости газов и каучуков.

\section{Упругость идеального газа}

Рассмотрим один моль идеального газа, находящегося в цилиндре с поршнем, при двух состояниях: $\mathrm{p}_{1}, \mathrm{~V}_{1}$ и $\mathrm{p}_{2}, \mathrm{~V}_{2}$, причем $\mathrm{p}_{1}>\mathrm{p}_{2}$ и $\mathrm{V}_{1}<\mathrm{V}_{2}$ [6].

Из опыта известно, что самопроизвольно газ может только расширяться, следовательно, состояние 2 термодинамически более выгодно. Причина этого заключается в том, что более разряженное состояние газа характеризуется большей термодинамической вероятностью и, следовательно, большей энтропией. Для расчета термодинамической вероятности используем простейшую объемную решетку, полагая, что в каждой ее ячейке объемом а ${ }^{3}$ ( $a$ - длина ребра кубической ячейки) находится одна молекула газа. Тогда для состояния 1 число способов расположения $N$ молекул газа в объеме $\mathrm{V}_{1}$ трактуемое в данном случае как термодинамическая вероятность, равно $\left(\mathrm{V}_{1} / a^{3}\right)^{\mathrm{N}}$, а для состояния $2-\left(\mathrm{V}_{2} / a^{3}\right)^{\mathrm{N}}$. Следовательно, относительное изменение термодинамической вероятности газа при переходе из состояния 1 в состояние 2 можно оценить отношением:

$$
\frac{W_{2}}{W_{1}}=\left(\frac{V_{2}}{V_{1}}\right)^{N} \text {. }
$$

Поскольку $\mathrm{V}_{2} / \mathrm{V}_{1}>1$ и $N>1$, то число $\mathrm{W}_{2} / \mathrm{W}_{1}$ очень велико, например, при $\mathrm{V}_{2} / \mathrm{V}_{1}=1,01, \mathrm{~W}_{2} / \mathrm{W}_{1}=1,01^{6 \cdot 10^{23}}$.

Таким образом, чтобы деформировать (сжать) газ, нужно увеличить давление, т.е. приложить силу. После прекращения воздействия газ самопроизвольно расширится до исходного состояния, которому отвечают большая термодинамическая вероятность и, следовательно, энтропия. Если при фиксированном объеме газ нагревать, то его давление увеличится. В результате для его сжатия следует приложить большее усилие, что означает увеличение модуля упругости газа с ростом температуры.

\section{Упругость идеального клубка}

Первая теория эластичности каучука, так называемая кинетическая теория, была предложена в 1932 г. швейцарским ученым Мейером, далее она получила развитие и подтверждение в работах Марка, Джоуля, Куна. В этой теории предполагается, что энтропия каучука складывается аддитивно, исходя из энтропии отдельных цепей. Этот принцип позволяет, учитывая молекулярно-кинетическое движение сегментов макромолекул, сразу же выявить причину обратимости высокоэластической деформации в каучуках.

Как термодинамическая система, изолированный макромолекулярный клубок напоминает газовое облако, в котором роль молекул выполняют кинетически не зависимые отрезки цепи - сегменты. Самопроизвольное тепловое движение сегментов не меняет внутренней энергии системы, поэтому

$$
F \equiv-T S \text {. }
$$


Энтропию идеальной цепи можно вычислить, исходя из уравнения Больцмана:

$$
S=k \cdot \ln W,
$$

где $W$ - термодинамическая вероятность.

В данном случае речь идет о конформационной энтропии, т.е. энтропии, связанной с возможностью реализации клубком множества конформаций.

Величина $W$ пропорциональна числу конформаций, возможных при заданном $R$. Поэтому $W \sim P_{(R)}$. Учитывая это, имеем:

$$
S=\text { const }-\frac{3}{2} k \frac{R^{2}}{\left\langle R^{2}\right\rangle} \text {. }
$$

Подставляя (7) в (5), получаем:

$$
F=\text { const }+\frac{3}{2} k T \frac{R^{2}}{\left\langle R^{2}\right\rangle} .
$$

Растяжение клубка под действием внешней силы приводит к отклонению $R$ от наиболее вероятной величины $\left\langle R^{2}\right\rangle^{1 / 2}$, уменьшению числа возможных конформаций и, следовательно, уменьшению энтропии.

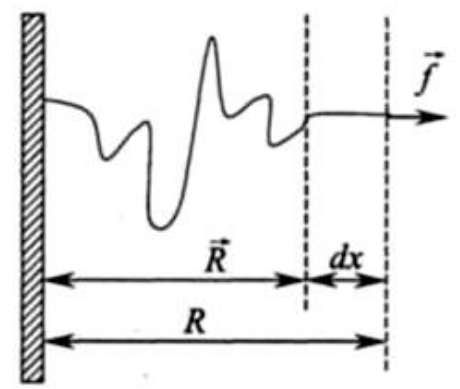

$a$

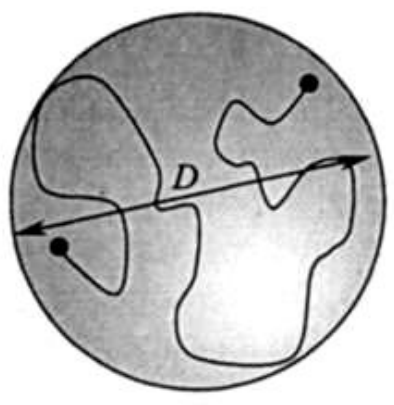

6

Рисунок 6 - Модель деформированного клубка: a - растяжение, б - сжатие.

Последнее прямо следует из формулы (7). В результате, возникает упругая сила, противодействующая растягивающей и стремящаяся вернуть клубок к состоянию с исходным $\left\langle R^{2}\right\rangle^{1 / 2}$ и максимумом энтропии. Выражение для величины упругой силы может быть получено, исходя из следующих соображений. Допустим, что один конец цепи закреплен, а к другому приложена сила $f$.

Под действием этой силы конец цепи стремится на расстояние $d x$, дальнейшему смещению будет препятствовать упругая сила $-f$, равная по величине, но противоположная по направлению приложенной силе. Поскольку при $V=$ const, $F=$ $d f / d x$ и в данном случае $d x=d R$, то 


$$
f=-\frac{T d S}{d R}=3 k T \frac{R}{\left\langle R^{2}\right\rangle}
$$

В рассматриваемой модели векторы $\vec{f}$ и $\vec{R}$ параллельны. Поэтому отношение $\mathrm{R} /\left\langle R^{2}\right\rangle$ можно рассматривать как относительную деформацию, и тогда уравнение (8) по содержанию становится аналогичным уравнению Гука. Из этой аналогии следует, что модуль упругости изолированного идеального клубка пропорционален $3 k T$, следовательно, он увеличивается с повышением температуры. Такое поведение также характерно для идеального газа. При сжатии клубка изменение функции Гиббса удобнее оценивать, пользуясь другой моделью. Рассмотрим идеальный гауссов клубок, содержащий п звеньев, помещенный внутрь непроницаемой для него сферы с диаметром $D$, причем

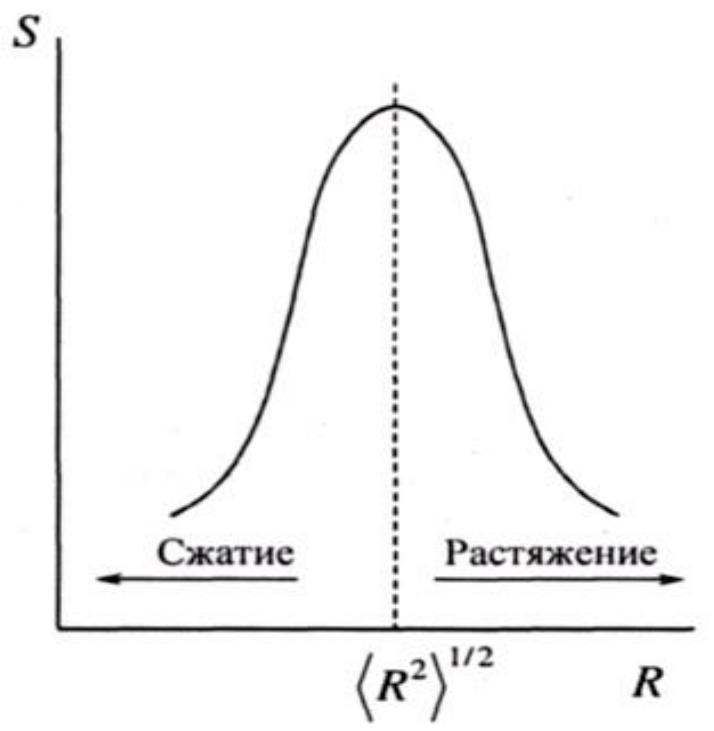

Рисунок 7 - Энтропия изолированного макромолекулярного клубка от его paзмера.

$D<\left\langle R^{2}\right\rangle^{1 / 2}$ (рис. 6, б). Очевидно, что в таких условиях цепь будет касаться стенок сферы в нескольких точках. Пусть средний отрезок цепи, заключенный между двумя контактами со стенкой, содержит в среднем $n^{*}$ звеньев. Тогда, очевидно, что число контактов клубка со сферой равно

$$
n / n^{*}
$$

и на каждом из этих контактов макромолекулярный клубок теряет половину своего конформационного набора. Следовательно, изменение энтропии, вызванное сжатием клубка в сфере, исходя из формулы Больцмана, будет равно:

$$
d S=S-S_{0}=-k \cdot \ln 2^{n / n^{*}},
$$

где $\mathrm{S}$ и $\mathrm{S}_{0}$ - энтропия деформированного и невозмущенного клубка. 
Таким образом, как растяжение, так и сжатие клубка приводят к уменьшению энтропии и возникновению упругой силы, которая стремится вернуть систему к исходному состоянию с максимумом энтропии, соответствующему среднеквадратичному размеру недеформированного клубка.

\section{Упругость полимерной сетки}

Чрезвычайно важное в практическом отношении свойство эластичности материально реализуется в резинах, т. е. сшитых каучуках, которые мы далее будем называть полимерными сетками. При теоретическом рассмотрении свойств полимерных сеток в условиях, когда реализуется подвижность сегментов (концентрированные растворы, гели, эластомеры), исходят из того, что отрезок цепи между двумя соседними сшивками, называемый субцепью, сворачивается в клубок, называемый субклубком, свойства которого аналогичны свойствам невозмущенного гауссового клубка. Такая модель позволяет качественно объяснить природу упругости резин аналогично тому, как это было сделано в предыдущем случае, т.е. как энтропийную. При действии приложенного напряжения, например растягивающего, размеры субклубка увеличиваются, что вызывает возникновение упругой силы, стремящейся вернуть клубки к исходному состоянию. По прекращении воздействия субклубки возвращаются к исходным размерам, при этом энтропия достигает максимально возможного значения [6].

Для количественного описания упругости полимерной сетки представим ее определенный объем в виде параллелепипеда, стороны которого ориентированы вдоль осей координат (рис. 8).

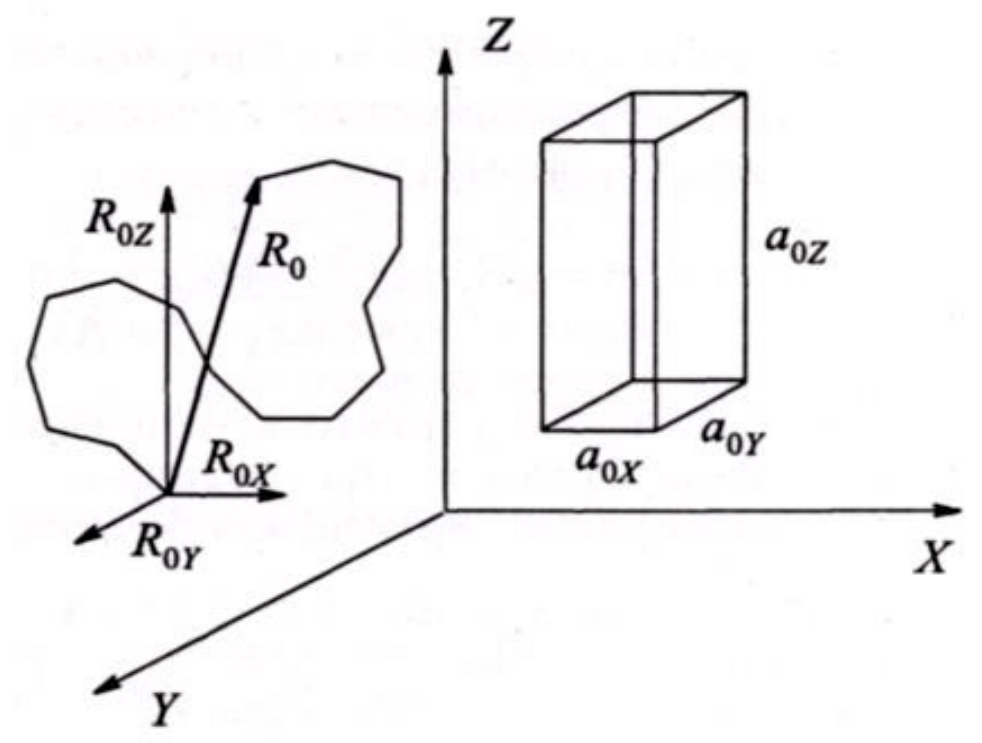

Рисунок 8 - Упругость полимерной сетки.

При растяжении сетки ее размеры вдоль каждой из осей $a_{0_{x}}, a_{0_{y}}, a_{0_{z}}$ изменяются соответственно в $\lambda_{\mathrm{x}}, \lambda_{\mathrm{y}}, \lambda_{\mathrm{z}}$ раз:

$$
a_{X}=a_{0 X} \cdot \lambda_{X}, a_{Y}=a_{0 Y} \cdot \lambda_{Y}, a_{Z}=a_{0 Z} \cdot \lambda_{Z} \text {, }
$$


где $\lambda_{x}, \lambda_{y}, \lambda_{z}$, - так называемые коэффициенты вытяжки. Изменение размеров сетки приводит к изменению размеров субклубков. Размер последних так же, как и в случае изолированной цепи, может быть охарактеризован радиусом-вектором, $\vec{R}_{0}$ который связан с его проекциями на оси координат соотношением:

$$
\vec{R}_{0}^{2}=\vec{R}_{0 X}^{2}+\vec{R}_{0 Y}^{2}+\vec{R}_{0 Z}^{2}
$$

При деформации сетки проекции $\mathrm{R}_{\mathrm{ox}}, \mathrm{R}_{0 \mathrm{Y}}, \mathrm{R}_{\mathrm{oz}}$ изменяются в такой же степени, как и линейные размеры сетки в направлении осей координат:

$$
\mathrm{R}_{\mathrm{x}}=\mathrm{R}_{\mathrm{ox}} \cdot \lambda_{\mathrm{x}}, \mathrm{R}_{\mathrm{y}}=\mathrm{R}_{\mathrm{oy}} \cdot \lambda_{\mathrm{y}}, \mathrm{R}_{\mathrm{z}}=\mathrm{R}_{\mathrm{oz}} \cdot \lambda_{\mathrm{z}}
$$

Изменение энтропии субклубка при изменении его размеров от $R_{0}$ до $R$, вызванном деформацией, можно учесть с помощью выражения (8), связывающего энтропию изолированного гауссового клубка с его размерами. В результате имеем:

$$
\begin{aligned}
\Delta S=S(R) & -S\left(R_{0}\right)=-\frac{3 k}{2 n l^{2}}\left[\left(R_{X}^{2}-R_{0 X}^{2}\right)+\left(R_{Y}^{2}-R_{0 Y}^{2}\right)+\left(R_{Z}^{2}-R_{0 Z}^{2}\right)\right]= \\
= & -\frac{3 k}{2 n l^{2}}\left[\left(\lambda_{X}^{2}-1\right) R_{0 X}^{2}+\left(\lambda_{Y}^{2}-1\right) R_{0 Y}^{2}+\left(\lambda_{Z}^{2}-1\right) R_{0 Z}^{2}\right],
\end{aligned}
$$

где $n$ - число звеньев в субцепи, $l$ - длина звена. Далее необходимо перейти от отдельной субцепи к полимерной сетке. Для этого выражение (10) необходимо умножить на число субцепей в единице объема $v$, равное количеству сшивок, и на общий объем полимерной сетки $V$. Кроме того, нужно учесть, что все направления равновероятны, и поэтому, с учетом (9), $R_{0 x}^{2}=R_{0 y}^{2}=R_{0 z}^{2}=\frac{n l^{2}}{3}$. Тогда для полимерной сетки в целом

$$
\Delta S=-k v V \frac{\lambda_{X}^{2}+\lambda_{Y}^{2}+\lambda_{Z}^{2}-3}{2}
$$

На практике наиболее часто встречается одноосное растяжение или сжатие, например вдоль оси $X$. Связанные с этим возможности изменения размеров сетки вдоль других осей легко установить, исходя из того фундаментального факта, что каучук и резина при деформации не изменяют объема. Отсюда легко рассчитать, что если $\lambda_{\mathrm{x}}=\lambda$, то

$$
\lambda_{\mathrm{y}}=\lambda_{\mathrm{z}}=\lambda^{-1 / 2}
$$

После подстановки этих значений в уравнение (11) получаем:

$$
\Delta S=-k v V \frac{\lambda^{2}+(2 / \lambda)-3}{2} .
$$

Ранее было показано, что при растяжении гауссовой цепи $\Delta F=-T \cdot \Delta S, f=$ $\partial F / \partial R$. Применительно к рассматриваемой системе упругая сила может быть представлена выражением: 


$$
f=-\frac{T \cdot \Delta S}{\Delta a_{X}}=-\frac{T \cdot \Delta S}{\Delta \lambda \cdot a_{0 X}}=-\frac{T \cdot S_{\lambda}^{\prime}}{a_{0 X}} .
$$

Для того, чтобы перейти к напряжению, необходимо разделить прилагаемую силу, равную по величине, но противоположную по знаку (направлению) силе упругости, на площадь образца:

$$
\sigma=\frac{f}{a_{0 Y} \cdot a_{0 Z}}=\frac{T \cdot S_{\lambda}^{\prime}}{a_{0 X} \cdot a_{0 Y} \cdot a_{0 Z}}=\frac{T \cdot S_{\lambda}^{\prime}}{V} .
$$

Раскрывая значение производной $S_{\lambda}^{\prime}$ окончательно имеем:

$$
\sigma=k T v\left(\lambda-\lambda^{-2}\right)
$$

Соотношение (15) является одним из главных результатов теории эластичности полимерных сеток.

Выражение для модуля упругости может быть получено из (15) для области малых деформаций, когда можно приближенно принять

$$
\lambda-\lambda^{-2}=(\lambda-1)+\left(1-\lambda^{-1}\right)\left(1+\lambda^{-1}\right) \approx(\lambda-1)+\frac{2(\lambda-1)}{\lambda} \approx 3(\lambda-1),
$$

что ведет к

$$
\sigma=3 k T v(\lambda-1) \text {. }
$$

Величина $(\lambda-1)=\frac{a_{x}-a_{0 x}}{a_{0 x}}$ является относительным удлинением, следовательно, модуль упругости полимерной сетки равен

$$
E=3 k T v,
$$

где $v$ - количество сшивок в единице объема.

Из данного уравнения следует:

- упругость полимерной сетки, определяемая величиной модуля, пропорциональна количеству сшивок в единице объема;

- модуль упругости полимерной сетки повышается с увеличением температуры.

Таким образом, из рассмотренного следует, что газы и каучуки, в том числе и «сшитые» (резины), имеют сходные характеристики упругости - их модули упругости 
близки и в обоих случаях повышаются с увеличением температуры; кроме того, известно, что сжатие приводит к повышению температуры как газов, так и каучуков. Эта аналогия объясняется одинаковой природой упругости, которая, с одной стороны, может быть охарактеризована как энтропийная, с другой - как молекулярнокинетическая. Первое указывает на природу обратимости деформации, второе - на способ ее реализации, который связан с перемещением молекул газа и сегментов молекул.

\section{Проблема вязкости полимерных систем}

Характерной особенностью полимерного состояния вещества является то, что все полимерные материалы, а также расплавы и концентрированные растворы полимеров обладают свойствами как упругого тела, так и жидкости. Это общее явление определяется как вязкоупругость, оно ответственно за особый комплекс свойств, присущих полимерным материалам.

Упруговязкая жидкость, т.е. жидкость, при течении которой накапливаются упругие (обратимые) деформации, может быть представлена механической моделью Максвелла.

К упруговязким жидкостям относятся достаточно концентрированные растворы полимеров, жидкие каучуки, расплавы полимеров. Рассмотрим простой опыт, который доказывает наличие упругого последействия в подобных жидкостях.

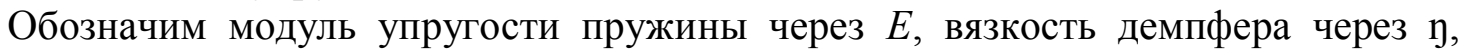
суммарное напряжение на пружине и поршне - через $\sigma$. Тогда скорость развития деформации $\varepsilon$ можно описать следующим соотношением:

$$
\frac{d \varepsilon}{d t}=\frac{d\left(\varepsilon_{\text {yпр }}+\varepsilon_{\text {тек }}\right)}{d t}=\frac{1}{E} \frac{d \sigma}{d t}+\frac{\sigma}{\eta}
$$

Рассмотрим случай, когда деформация модели фиксируется, т.е. $\varepsilon=$ const и напряжение начнет постепенно уменьшаться в результате возвращения пружины к исходному состоянию. Процесс этот является замедленным, поскольку связан с перемещением поршня в вязкой жидкости. Если в начальный момент, отвечающий фиксации деформации, $t=0, \varepsilon=\varepsilon_{0}>\sigma=\sigma_{0}$ и $d \varepsilon / d t=0$, то в результате интегрирования уравнения (16) получаем:

$$
\begin{gathered}
\sigma(t)=E \varepsilon_{0} \exp \left\{-\frac{t}{\tau}\right\} \\
\sigma(t)=\sigma_{0} \exp \left\{-\frac{t}{\tau}\right\}, \\
\tau=\eta / E .
\end{gathered}
$$

Параметр $\tau$ называется временем релаксации.

$\tau$ можно определить как время, в течение которого напряжение уменьшается в «е» раз. Согласно более общему определению, $\tau$ можно представить как время, 
необходимое для перестройки структуры системы, подвергающейся воздействию, из исходного равновесного состояния к конечному. Явление уменьшения напряжения при фиксированной деформации упруговязкого тела называется релаксацией напряжения.

С помощью методов квантовой химии можно рассчитать многие свойства молекул с любой точностью. То есть можно рассчитать такие свойства как геометрические параметры молекул, определяющие равновесную структуру молекулы, потенциальные поверхности, электронные спектры, энергии разрыва межатомных связей и т.д. Однако чаще всего для понимания какого-либо процесса интересно поведение не индивидуальных молекул, а молекулярных ансамблей, то есть коллективные (коллигативные) свойства, например температура плавления или стеклования вещества, его плотность, диффузионные характеристики. Под ансамблем в зависимости от задачи можно понимать как несколько десятков молекул, организованных в виде кластера, так и макроскопическую совокупность, в которой количество молекул соответствует числу Авогадро $\mathrm{N}_{\mathrm{A}}=6,023 \cdot 10^{23}$. В настоящее время методами квантовой химии можно делать достаточно надежные расчеты лишь для молекулярных систем с числом атомов до одной-двух сотен. Как видно этого явно недостаточно для предсказания коллективных свойств или характеристик даже отдельно взятых макромолекул. Кроме того, следует иметь в виду, что полимеры всегда находятся в окружении плотной среды - низкомолекулярного растворителя или других макромолекул. И это окружение также необходимо принимать во внимание.

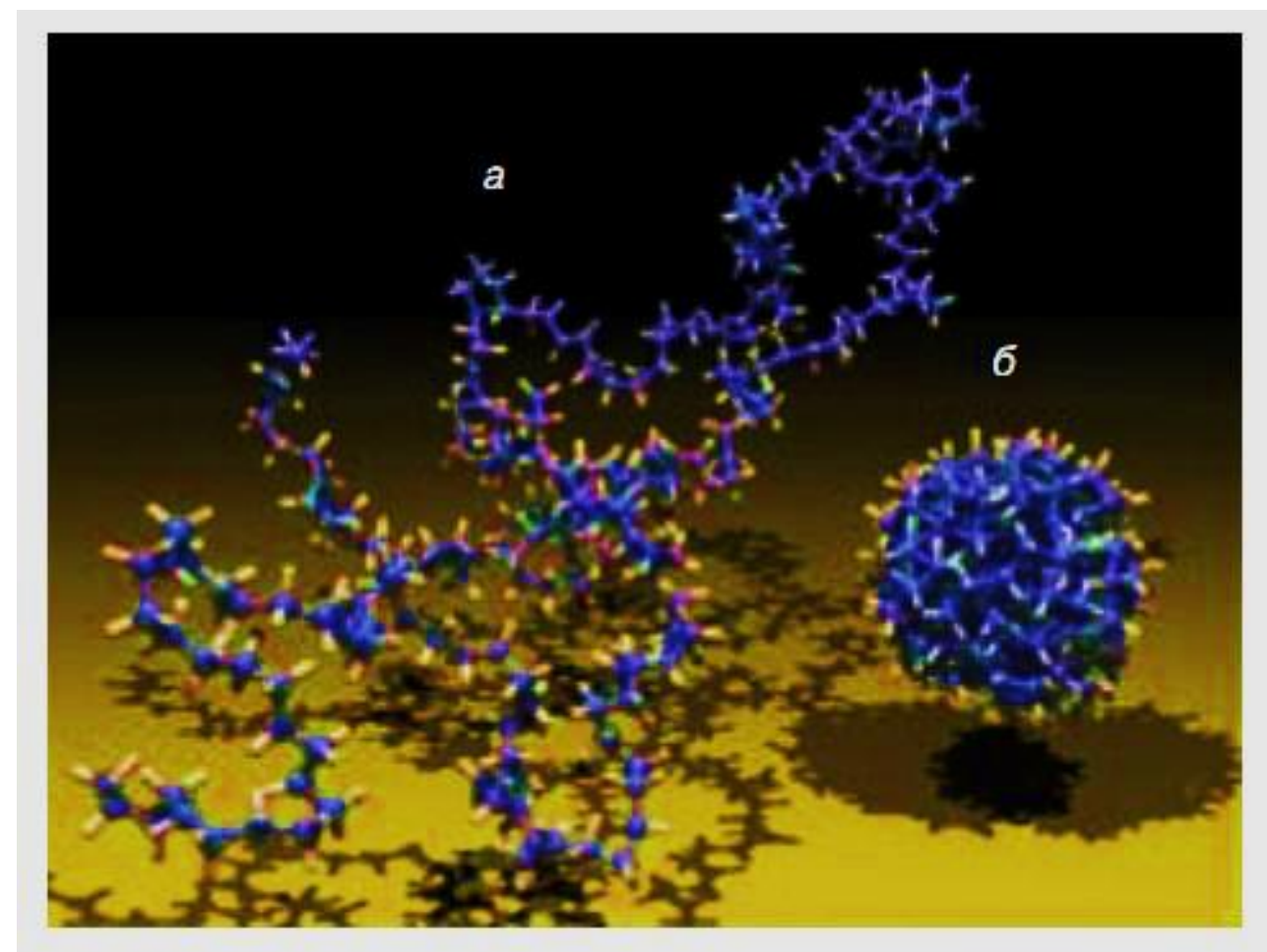

\section{Рисунок 9 - Развернутая и сжатая конформации 1000-звенной полиэтиленовой цепи, полученные при моделировании методом Монте-Карло [4].}

В компьютерном моделировании, как уже говорилось выше, используется язык классической физики. Этот отказ от рассмотрения молекулы как электронно-ядерной системы, позволяет коренным образом расширить круг явлений и объектов, доступных исследованию в компьютерном эксперименте. При расчетах методами Монте-Карло или молекулярной динамики существующие суперкомпьютеры способны оперировать системами, в которых число частиц достигает нескольких миллионов. Даже на 
современных персональных компьютерах легко удается моделировать многие коллективные свойства различных систем, включая полимерные растворы и расплавы.

Все термодинамические свойства любой системы зависят от температуры, но изучение зависимости их от состава и строения фаз является важной задачей физической химии. Наиболее важные из них это давление, коэффициент теплового расширения, параметры фазовых равновесий. Квантовая химия описывает молекулы при абсолютном нуле температуры. Влияние теплового движения атомов должно учитываться с помощью иных теоретических подходов. В общем случае можно сказать, что за исключением лишь сравнительно небольшого числа идеализированных ситуаций, поведение любой реальной системы определяется ее свободной энергией. Эта энергия складывается из энергетического (энтальпийного) и энтропийного вкладов. Из них наиболее интересен последний. Он связан с числом конформаций, которое способна принимать индивидуальная молекула, и числом конфигураций всего молекулярного ансамбля. Наблюдаемые на опыте свойства всегда являются результатом усреднения по множеству различных состояний системы. Как правило, только такие свойства (например, среднеквадратичные размеры полимерного клубка, среднеквадратичный дипольный момент и поляризация, характеристики светорассеяния) представляют интерес для полимерных систем. Компьютерный эксперимент нацелен на получение именно такой информации и, следовательно, может быть использован для проведения соответствующих расчетов.

Наконец, существует обширная группа динамических, то есть зависящих от времени, характеристик. Они определяют диффузию, вязкость, коэффициенты седиментации, теплопроводность, отклики системы на внешние воздействия и др. Кроме того, для полимеров чрезвычайно важно знать реологические свойства, включающие поведение растворов и расплавов при течении, при сдвиговых деформациях и т.д. Однако время как параметр не входит в квантово-химические уравнения. Следовательно, если необходимо предсказание таких свойств исходя из молекулярных параметров, должен использоваться численный эксперимент динамического типа (включая методы неравновесной молекулярной динамики).

\section{Поворотно-изомерный механизм гибкости цепи.}

К гибкоцепным полимерам относятся полиолефины, большинство полимеров виниловых и винил-диеновых мономеров общей формулы -( $\left.\mathrm{CH}_{2}-\mathrm{CHX}\right)-,-\left(\mathrm{CH}_{2}-\mathrm{CXY}\right)-$, где $\mathrm{X}, \mathrm{Y}$-заместители основной цепи [6]. Гибкость таких полимеров обусловлена свободой вращения вокруг простых связей основной цепи, механизм гибкости называется поворотно-изомерным. Рассмотрим детали этого механизма на примере бутана, который можно представить как фрагмент цепи полиэтилена (рис. 10). При вращении связи $\mathrm{C}_{1}-\mathrm{C}_{2}$ или $\mathrm{C}_{3}-\mathrm{C}_{4}$ описывается конус с образующей, направленной под углом $\delta$ к оси вращения. Угол $\delta$ является дополнительным к валентному, т.е. $\delta=\pi-$ $190^{\circ}$. При вращении связи $\mathrm{C}_{3}-\mathrm{C}_{4}$ атом $\mathrm{C}_{4}$ описывает окружность, в плоскости которой лежит угол вращения $\gamma$, отсчитываемый относительно транс-положения. Расстояние между конечными атомами $\mathrm{C}_{1}$ и $\mathrm{C}_{4}$ при вращении изменяется и составляет, как показывает расчет, 0,2 нм для цис- и 0,38 для транс-формы. Поскольку радиусы Вандер-Ваальса метильной группы примерно равны 0,2 нм, можно ожидать стерического напряжения иис-формы. В этом случае вращение вокруг связи $\mathrm{C}_{2}-\mathrm{C}_{3}$ не будет свободным. Вследствие взаимного отталкивания заместителей в i/wc-положении возникают потенциальные барьеры вращения.[6] 

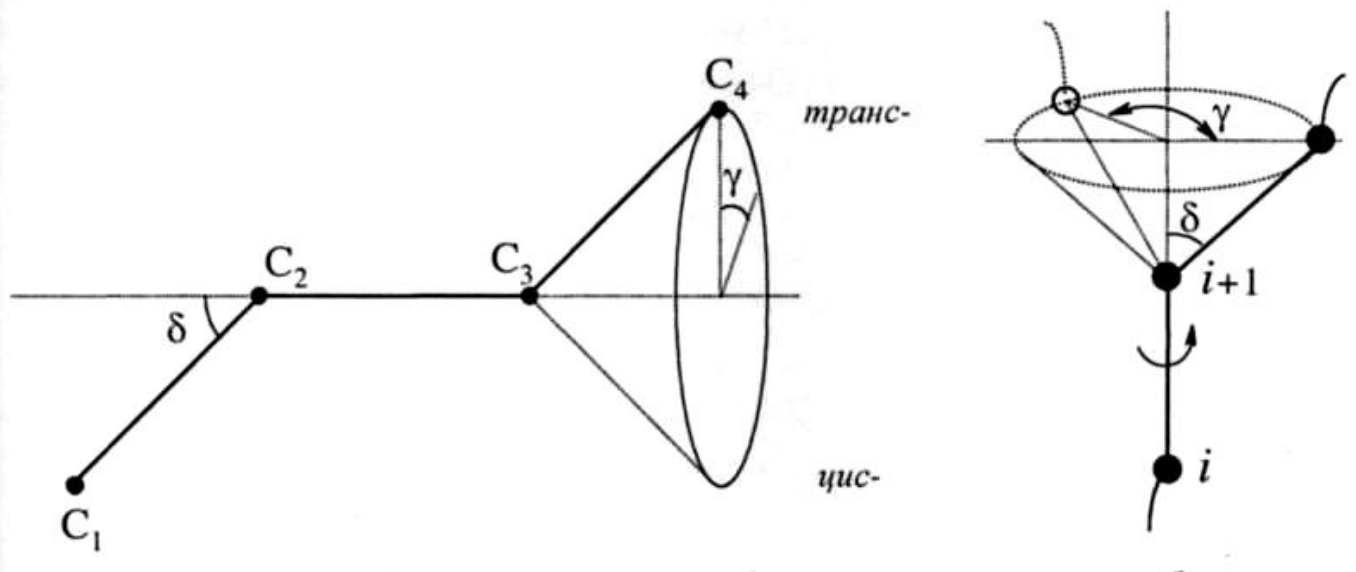

$a$

6

Рисунок 10 - Вращение атомоа вокруг связей.

Наличие заторможенного вращения вокруг связей C-C нормальных парафинов впервые было экспериментально обнаружено М.С.Ньюменом. По методу Ньюмена изменение формы молекул в результате вращения связей изображают проекциями связей на плоскость, нормальную к оси вращения (рис. 16).

Если смотреть вдоль оси С3-С2, то при вращении связи С3-С4 возможны заслоненные конформации, когда проекции связей на плоскость совпадают (цис- и цисгош), и заторможенные (скрещенные) конформации (транс- и транс-гош) (на рис. 2.15 заслоненные конформации обозначены скобками). При вращении вокруг связи $\mathrm{C}_{2}-\mathrm{C}_{3}$ потенциальная энергия системы периодически изменяется - транс-конформациям отвечают минимумы энергии, цис-конформациям - максимумы (рис. 16). Наиболее глубокий минимум отвечает транс-конформации, от которой отсчитывается угол $r$. При вращении по часовой стрелке гош-конформациям приписывается знак «+», при вращении против часовой стрелки - знак «-». Аналогично обозначение конформации, возникающих при вращении вокруг связей С-С основной цепи полимеров виниловых мономеров (рис. 10, б).[6]. Построим 3D модель и будем отображать не молекулы, а конусы по которым вращаются связи (рис.11-15).[7]

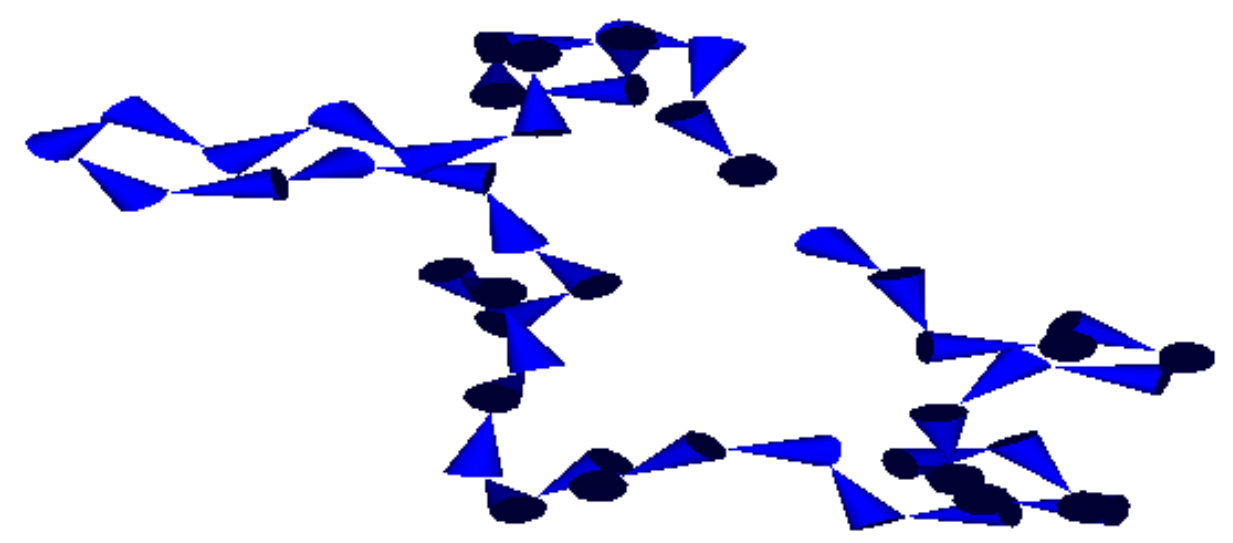

Рисунок 11 - Молекула полимера (50 звеньев), для размерности в 10 анг. 


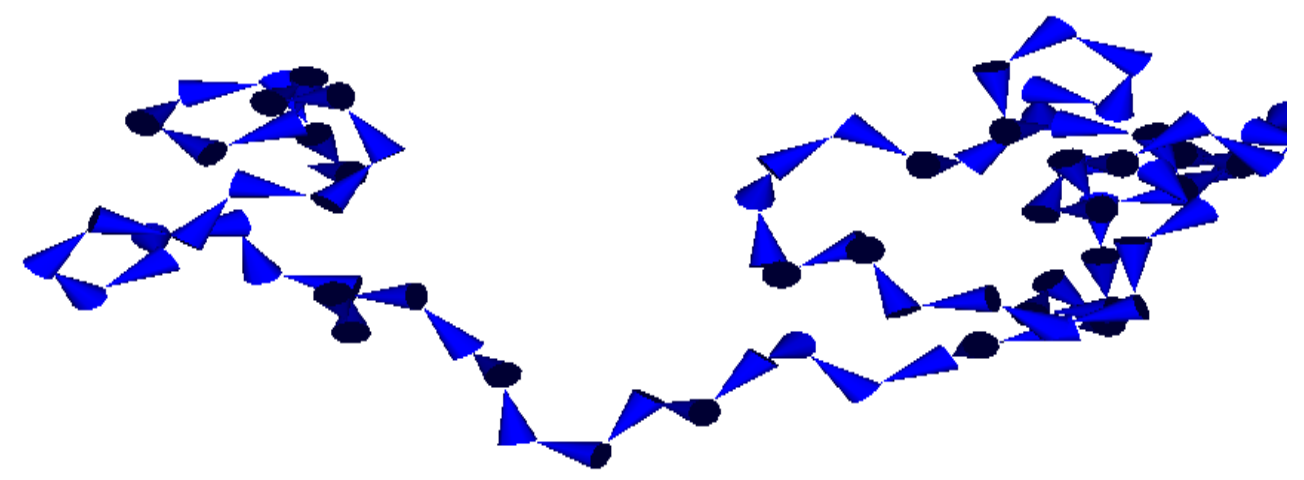

Рисунок 12 - Молекула полимера (50 звеньев), для размерности в 10 анг.
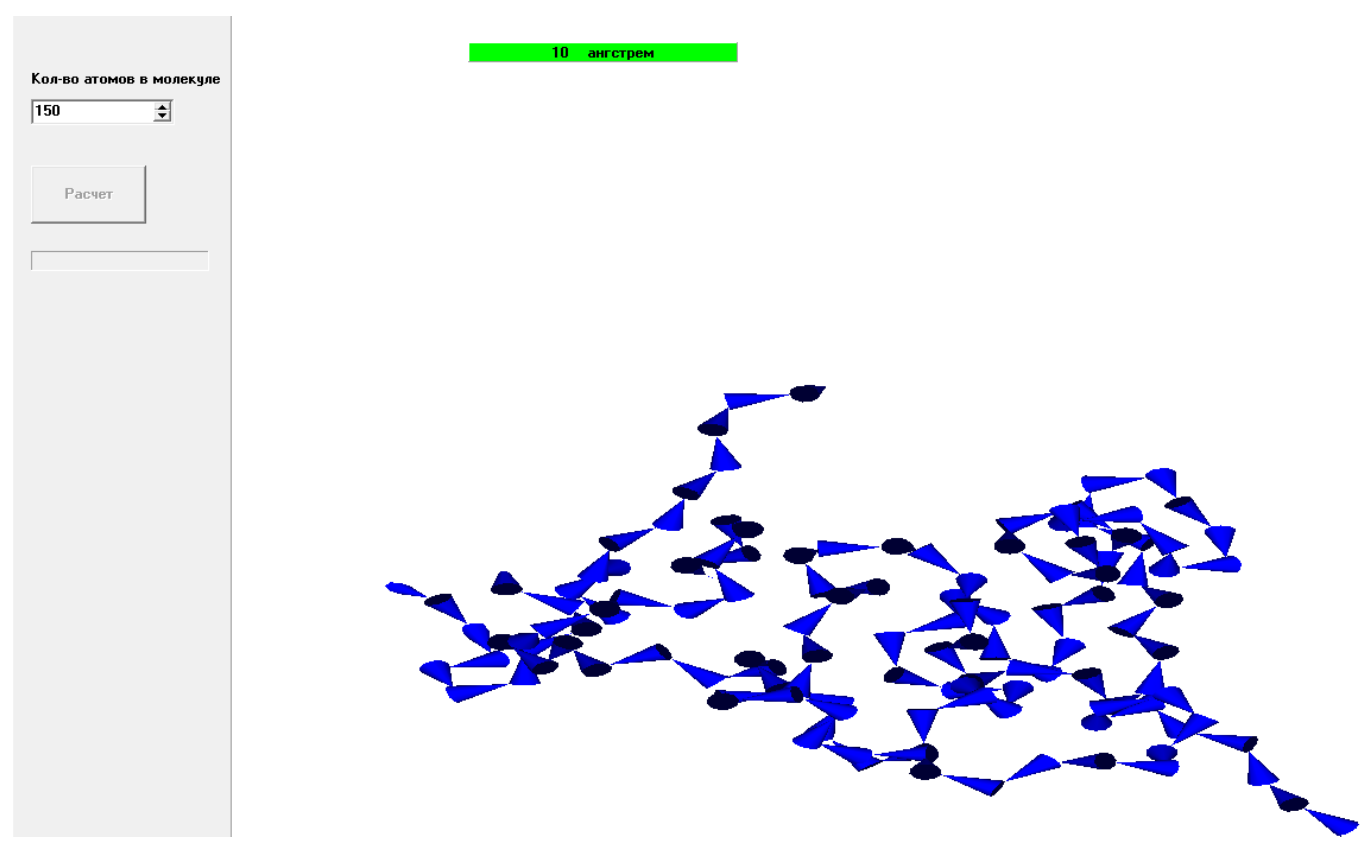

Рисунок 13 - Молекула полимера (100 звеньев), для размерности в 10 анг. 

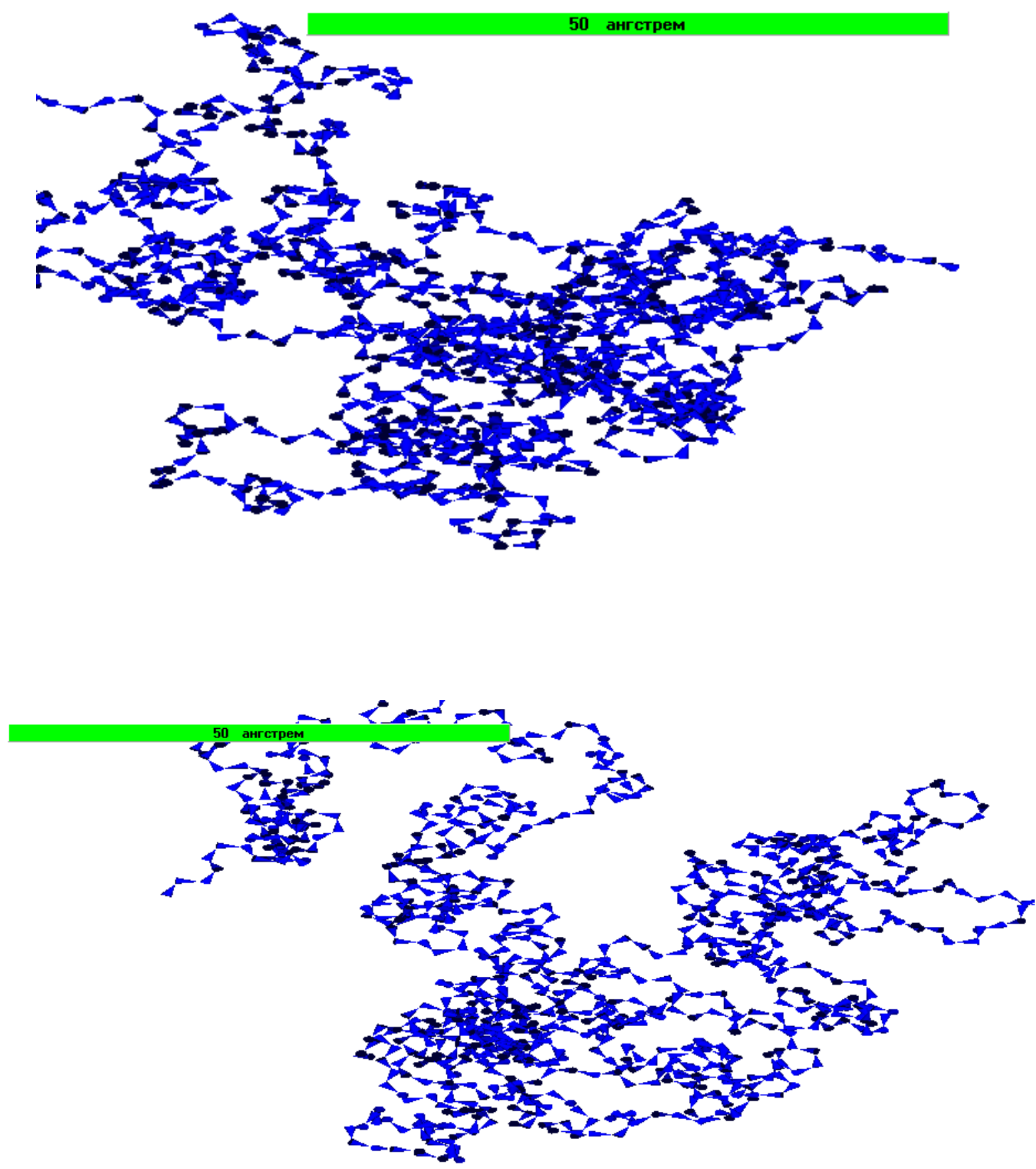

Рисунок 14 - Молекулы полимера (1000 звеньев), для размерности в 50 анг.

Разница между максимальной энергией, отвечающей заслоненной цисконформации, и минимальной энергией, отвечающей скрещенной транс-конформации в $н$-бутане, настолько значительна, что свободного полного вращения вокруг связи $\mathrm{C}_{2}-$ $\mathrm{C}_{3}$ не происходит. Молекула находится в одной из 

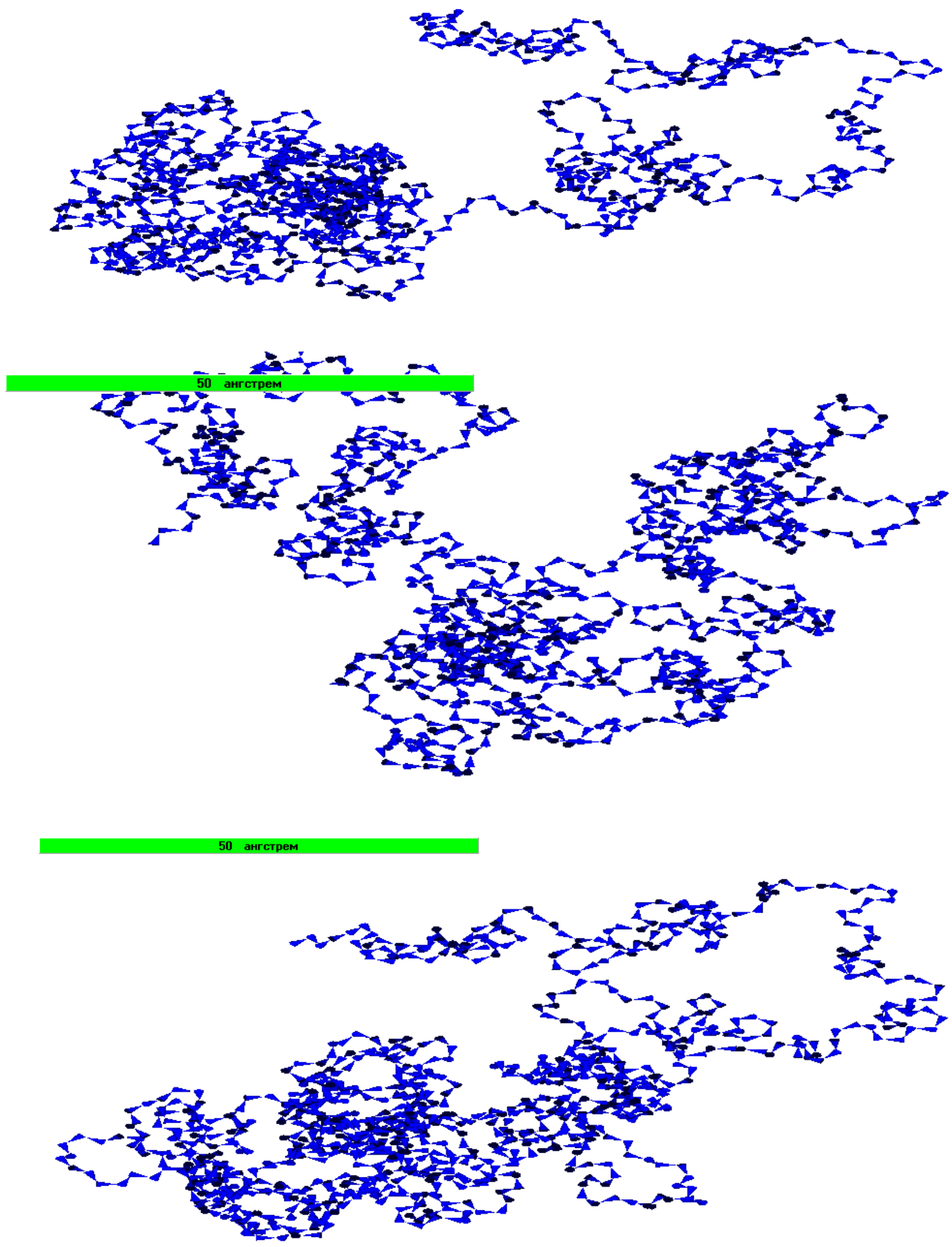

Рисунок 15 - Молекулы полимера (1000 звеньев), для размерности в 50 анг. 

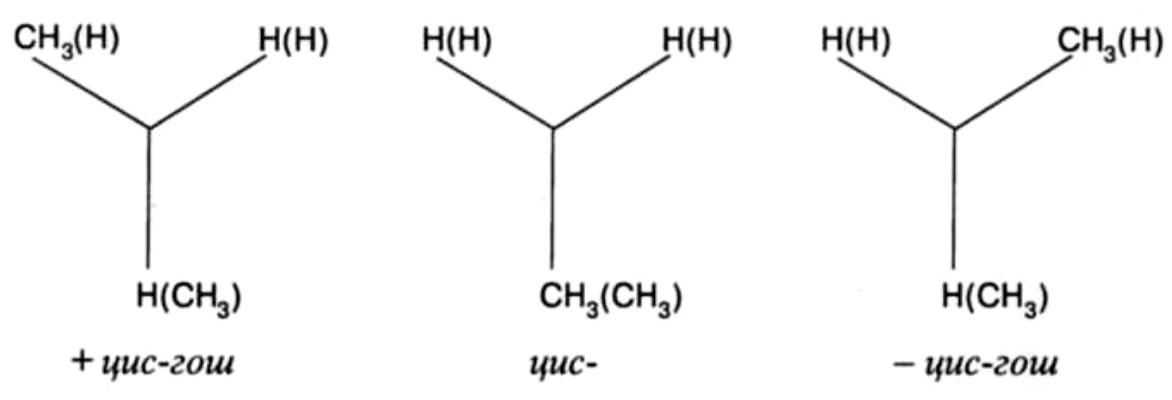

заслоненные конформации<smiles>CCC</smiles>

+ транс-гош<smiles>CCC</smiles>

транс-<smiles>CCC</smiles>

- транс-гош

скрещенные конформации

Рисунок 16 - Проекции Ньюмена.

конформаций с минимальными значениями энергии: транс-, -транс-гош и +транс-гош формах.

Энергии этих конформаций отличаются всего на 2,5 кДж/моль $(\Delta \varepsilon)$, а потенциальные барьеры $(\Delta E)$, разделяющие их, равны 14 кДж/моль, т.е. также относительно невелики, поэтому происходит постоянная смена конформаций в результате частичного неполного заторможенного вращения вокруг связи $\mathrm{C}_{2}-\mathrm{C}_{3}$.

Потенциальные барьеры заторможенного вращения получили название потенциалов торможения. [6] 

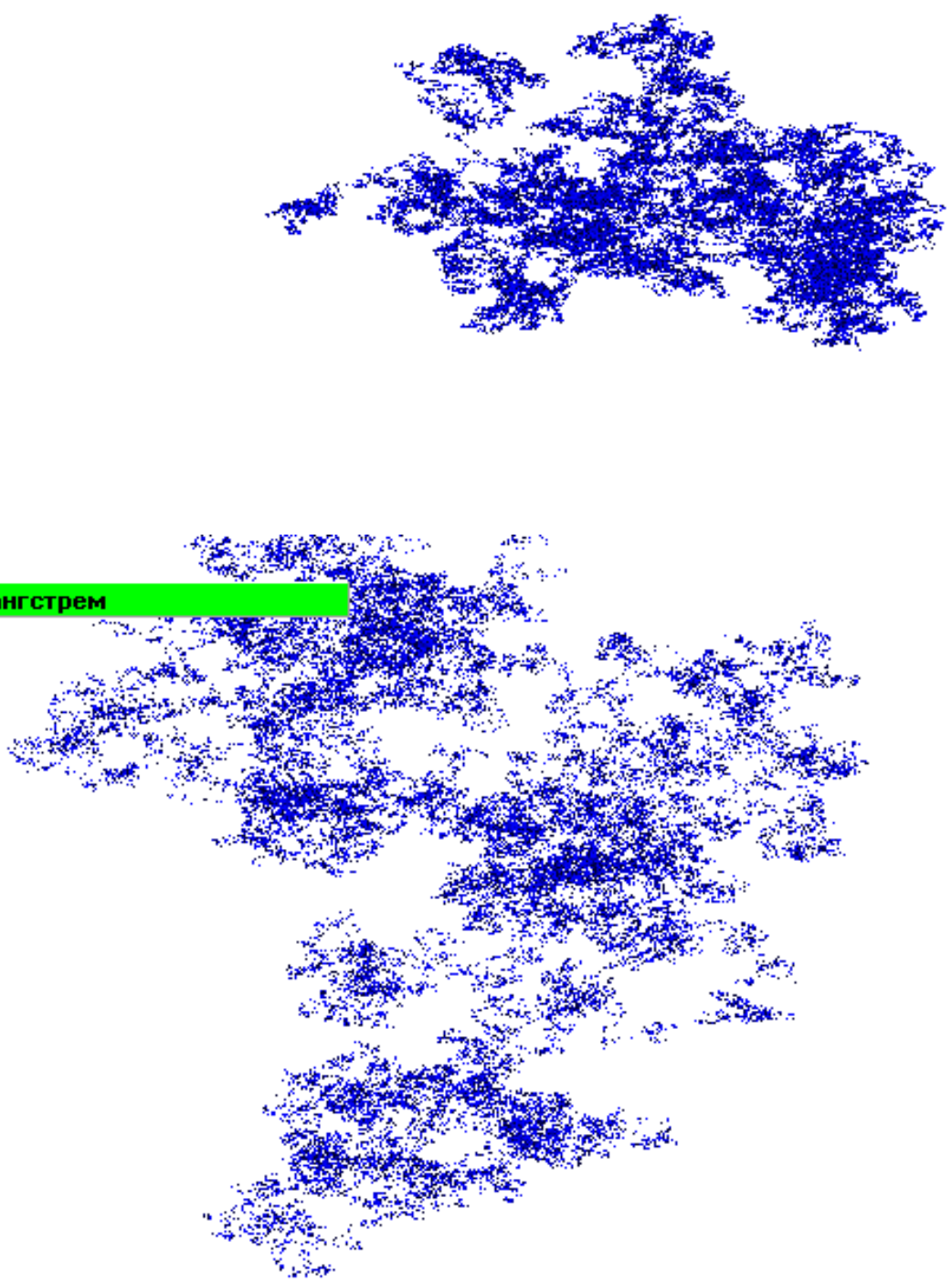

Рисунок 17 - Макро молекулы полимера (600 000 звеньев), для размерности в 1000 анг. 

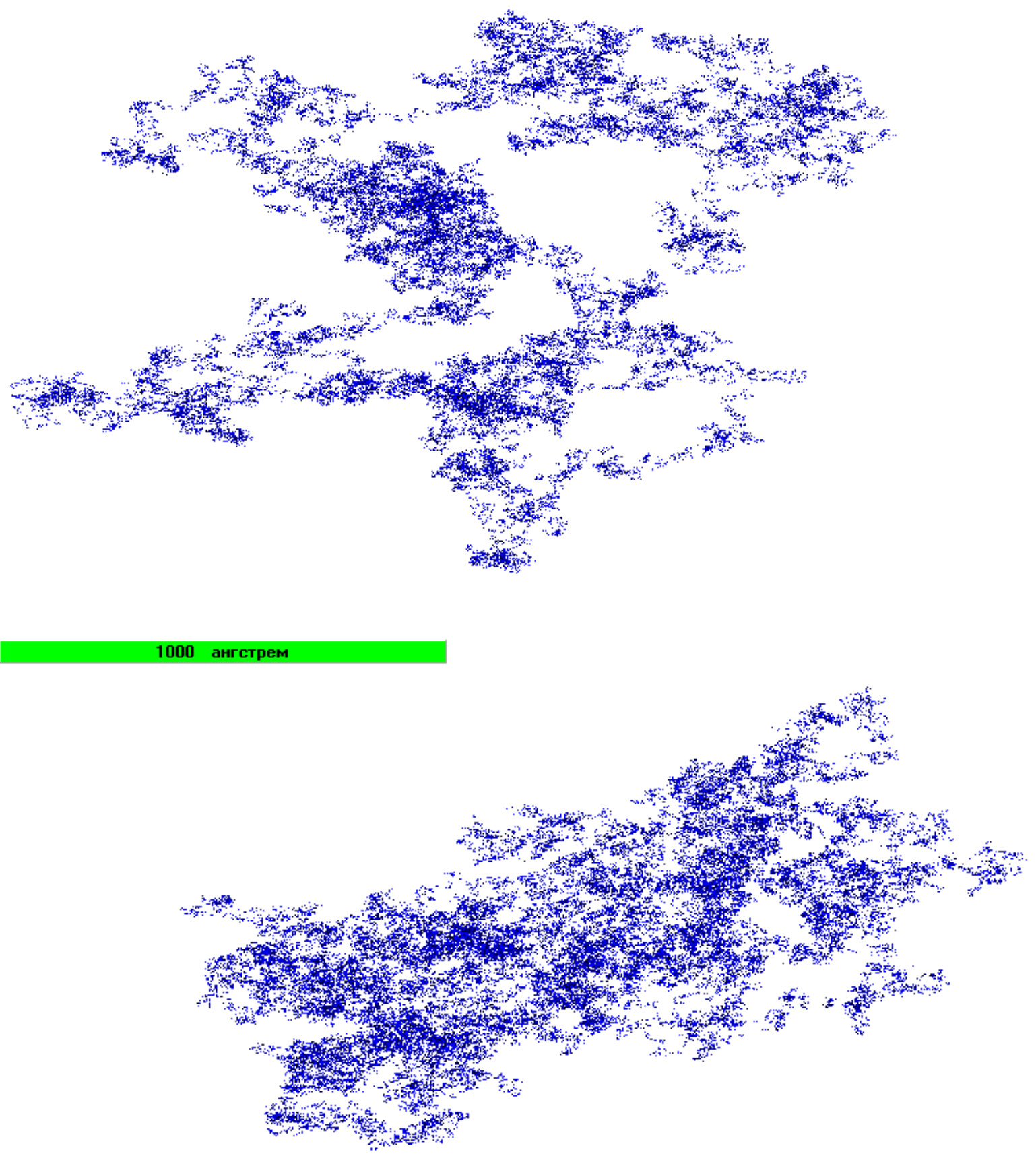

Рисунок 18 - Макро молекулы полимера (600 000 звеньев), для размерности в 1000 анг. 


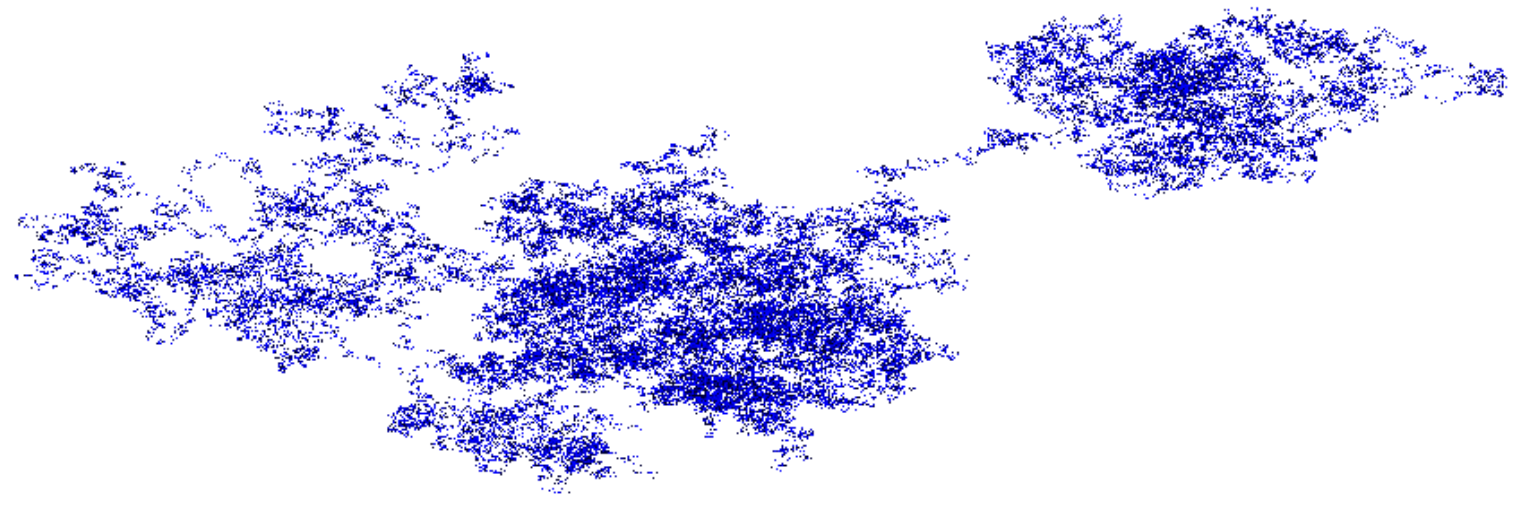

1000 ангстрем

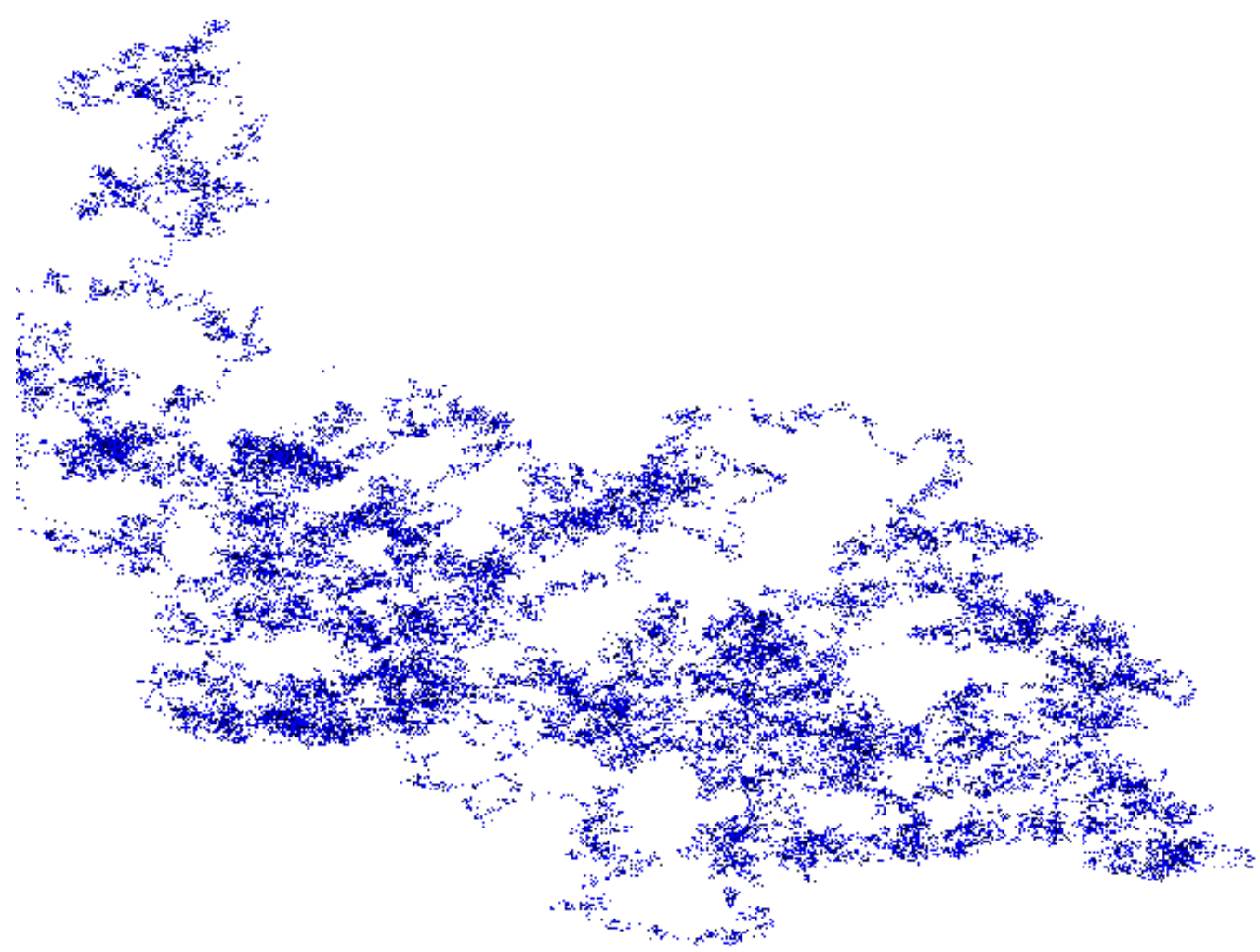

Рисунок 19 - Макро молекулы полимера (600 000 звеньев), для размерности в 1000 анг. 


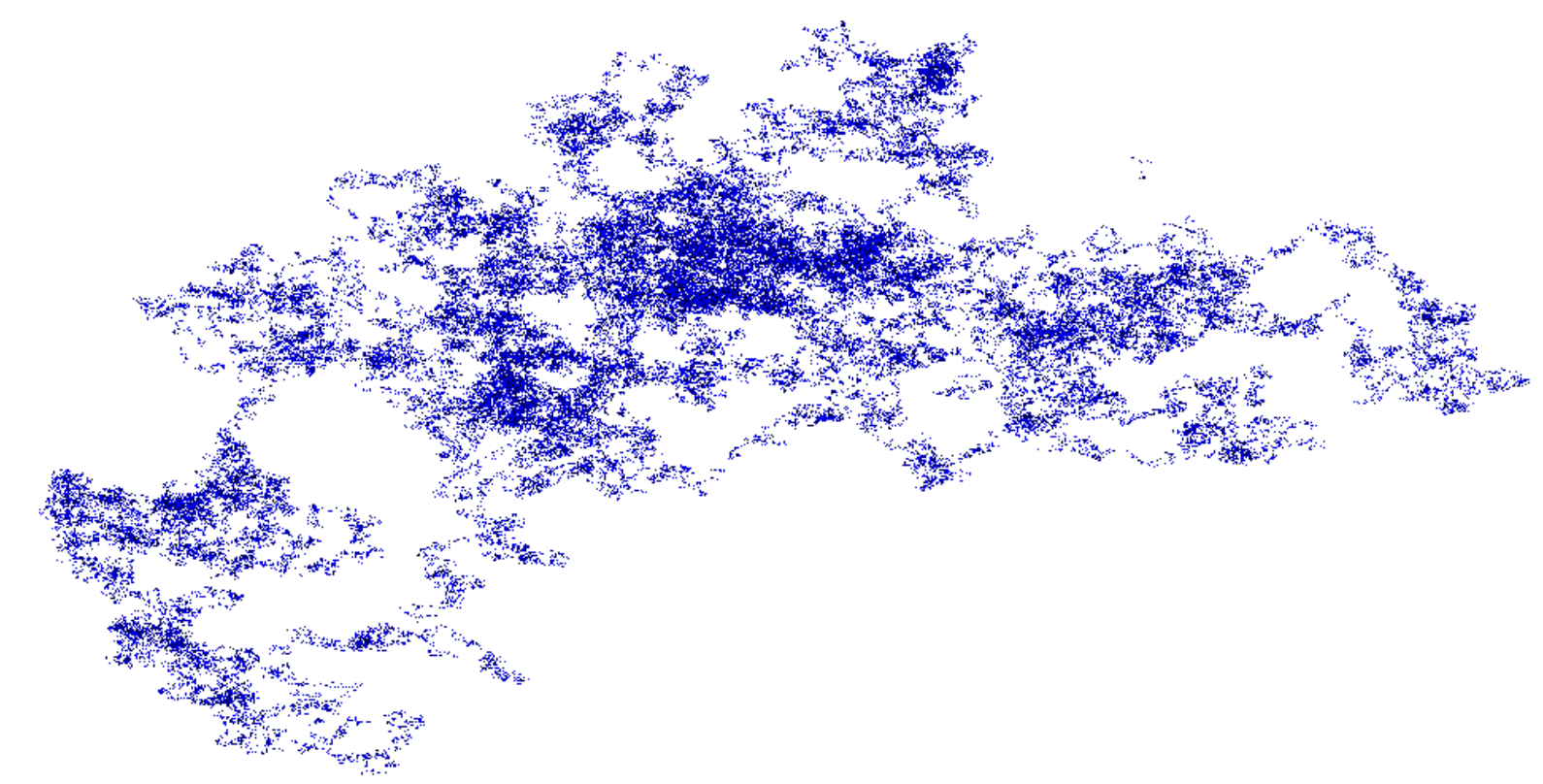

Рисунок 20 - Макро молекула полимера ( 1000000 звеньев), для размерности в 1000 анг.

\section{Выводы}

Современная наука не может рассматривать какую либо модель без ее компьютерной аппробации и достоинство методов компьютерного эксперимента принципиальная возможность строгого описания весьма широкого круга моделей. В то же время строгие аналитические решения могут быть получены только в редких случаях. Обычно же в аналитических теориях приходится прибегать к тем или иным упрощениям, таким, например, как линеаризация исходных уравнений, разложение в ряд по малому параметру, разделение переменных, сведение к задаче меньшей размерности, асимптотическое представление. Практически любые конденсированные многочастичные системы вызывают повышенные сложности для строгого аналитического описания. В этих условиях компьютерный эксперимент становится необходимым для проверки справедливости исходных приближений, заложенных в теорию, и логических следствий, вытекающих из аналитического рассмотрения.

\section{References:}

1. Компьютерное моделирование полимеров. [Электронный ресурс]. URL

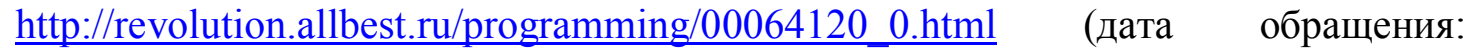
18.03.2014).

2. Пиотровская Е.М. Исследование различных вариантов решеточных моделей растворов методом Монте-Карло. диссертация кандидата химических наук : 02.00.04 Ленинград, 1974. -199 с. 
3. Набиуллин А.Р. Применение методов Монте-Карло и регуляризации Тихонова для моделирования начальной стадии радикальной полимеризации. Диссертация кандидата химических наук, Бирск, -131с.

4. Реферат - Холестерин - строение, биологическое значение, контроль содержания. Компьютерное моделирование полимеров [Электронный ресурс]. URL http://nashaucheba.ru/v27387/?download2=file (дата обращения: 18.03.2014).

5. Новиков В.В. Компьютерное моделирование полимеров. -Тверь, 2002. -21с.

6. Семчиков Ю. Д.СЗО Высокомолекулярные соединения: Учеб. для вузов. - Н. Новгород: Издательство Нижегородского государственного университета им. Н. И.Лобачевского; М.: Издательский центр «Академия», 2003. - 368 с.

7. Shevtsov A.N., Keulimzhayeva Z.A., Alpysbayev E.A. ABOUT SOME MODELS OF POLYMER MACROMOLECULES. ISJ Theoretical \& Applied Science. №11(7), 2013. ( ISPC «Applied scientific research», 30.11.2013, Belgrade, Serbia ). pp.26-40.

8. Shevtsov A.N., Alimbaev B.A., Manapbaev B.J. COMPUTER MODEL OF THE INFLUENCE OF MAGNETIC FIELDS OF THE DOMAINS IN THE FORM OF MICRO-CRACKS AT FRACTURE OF METALS. ISJ Theoretical \& Applied Science. - №6 (2), 2013. (ISPC, «World of Science», 30.06.2013, Hamburg, Germany). - pp.51-64.

9. Kestelman V.N., Jönsson A., Shevtsov A.N. ON SOME DEPENDENCIES STATISTICAL METHODS FOR MODELING OF LONG-TERM STRENGTH OF POLYMERS ISJ Theoretical \& Applied Science. - №7 (3), 2013. (ISPC Theoretical Research, 30.07.2013, Stuttgart, Germany). - pp.69-77.

10. Бартенев Г.М., Зеленев Ю.В. Физика и механика полимеров. -М.: Высшая школа, 1983, 390с.

11. Физика полимеров. ЛНУ им. Т.Шевченко, 2009. [Электронный ресурс]. URL http://softacademy.Inpu.edu.ua/Programs/ fizika polimerov/index.htm (дата обращения: 18.03.2014). 